\title{
Domain observations in Fe-Pd-Co by dynamic in-situ Lorentz TEM
}

\author{
A. Budruk ${ }^{1}$, C. Phatak ${ }^{2}$, A.K. Petford-Long ${ }^{2}$ and M. De Graef ${ }^{1}$ \\ ${ }^{1}$ Department of Materials Science and Engineering, Carnegie Mellon University, Pittsburgh PA 15213 \\ ${ }^{2}$ Argonne National Laboratory, 9700 Cass Avenue, Argonne, IL 60439
}

Understanding the interactions between magnetic domain walls and twin boundaries in multiferroic materials is of great significance, since such interactions can lead to interesting material properties; for example, the large magnetic field induced strain $(\approx 10 \%)$ observed in the ferromagnetic shape memory alloy $\mathrm{Ni}_{2} \mathrm{MnGa}[1]$ is due to the intricate interplay between magnetic domains and twin variants. The high spatial resolution achievable in dedicated Lorentz instruments is especially advantageous to study magnetic domains in materials with very fine microstructural features, such as martensitic twins. When combined with phase reconstruction algorithms, the Fresnel (of out-of-focus) mode of Lorentz TEM (LTEM) makes it possible to obtain a projection of the magnetization inside the sample [2]. Since the sample sits in a field-free region inside the microscope, LTEM can be used to study the domain structure in the demagnetized state. It is also possible to apply a magnetic field inside the microscope to study the dynamics of domain walls. In the past, such studies have been carried out by exciting the objective lens [3]. However, this method applies a field parallel to the electron beam. With the introduction of in-situ magnetizing holders, a field of known magnitude can be applied perpendicular to the electron beam and the domain structure evolution can be studied in both the demagnetized and magnetized states.

In the present study, an in-situ magnetizing holder designed by Hummingbird Scientific was used on a JEOL $2100 \mathrm{~F}$ dedicated Lorentz microscope operated at $200 \mathrm{kV}$ to study domain wall dynamics in the martensitic state of a $\mathrm{Fe}_{68} \mathrm{Pd}_{30} \mathrm{Co}_{2}$ alloy. Under zero applied field, two different types of domain walls were observed (Fig. 1): 1) walls that coincide with the twin boundaries of the underlying martensitic microstructure and 2) walls that run across the twin boundaries. Under the application of the magnetic field, the domain walls of the second kind were observed to move first. An applied magnetic field of just over 10 Oe was sufficient to move these walls, which were not pinned by the twin boundaries. An applied field of more than 300 Oe, however, was insufficient to move the walls coinciding with the martensitic twins. The changing magnetization distribution with applied magnetic field was determined from phase reconstruction results (Fig. 2), which clearly indicate that the domains that are unfavourably oriented with respect to the applied magnetic field become smaller and are eventually eliminated. It was also observed that the domains in adjacent twin bands are related by a $90^{\circ}$ rotation of magnetization direction. It can be concluded that the magnetic domains inside the twin variants are strongly tied to the underlying twin structure, which explains the strong pinning effect of twin boundaries on the magnetic domain walls. Additional domain wall - twin boundary interactions will be described.

\section{References}

[1] A. Sozinov, A. A. Likhachev, N. Lanska, and K. Ullakko. Appl. Phys. Lett., 80:1746, 2002.

[2] M. De Graef. Lorentz Microscopy, Editors: M. De Graef and Y. Zhu. Vol. 36 of Experimental Methods in Materials Sciences, Chapter 2. Academic Press, 2000.

[3] Y. Murakami, D. Shindo, K. Oikawa, R. Kainuma, K. Ishida, Acta Materialia, 50:2173-2184, 2002.

[4] This work was supported by NSF-DMR \#0404836. Part of this work carried out at Argonne National Laboratory, a U.S. DOE Office of Science laboratory, operated under Contract No. DE-AC02-06CH11357. 

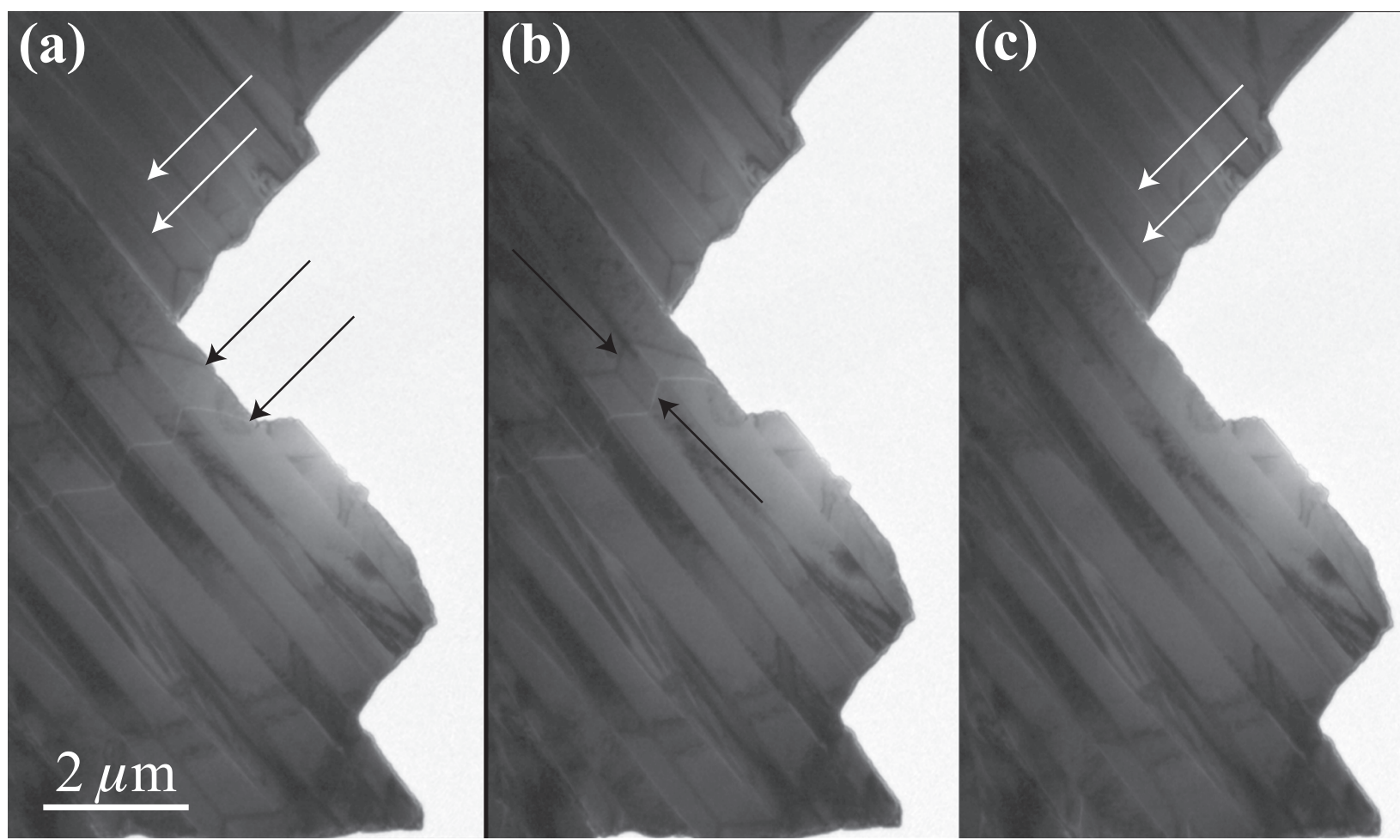

Figure 1: (a) Fresnel over-focus image of twinned microstructure in $\mathrm{Fe}_{68} \mathrm{Pd}_{30} \mathrm{Co}_{2}$ at zero applied field $H_{A}$. White arrows point to walls coinciding with twin boundaries; dark arrows indicate mobile walls. (b) The same region of the foil at $H_{A}=15 \mathrm{Oe}$. Black arrows indicate the direction of motion of domain walls. (c) At $H_{A}=23 \mathrm{Oe}$, walls pinned on twin boundaries still persist (indicated by white arrows).

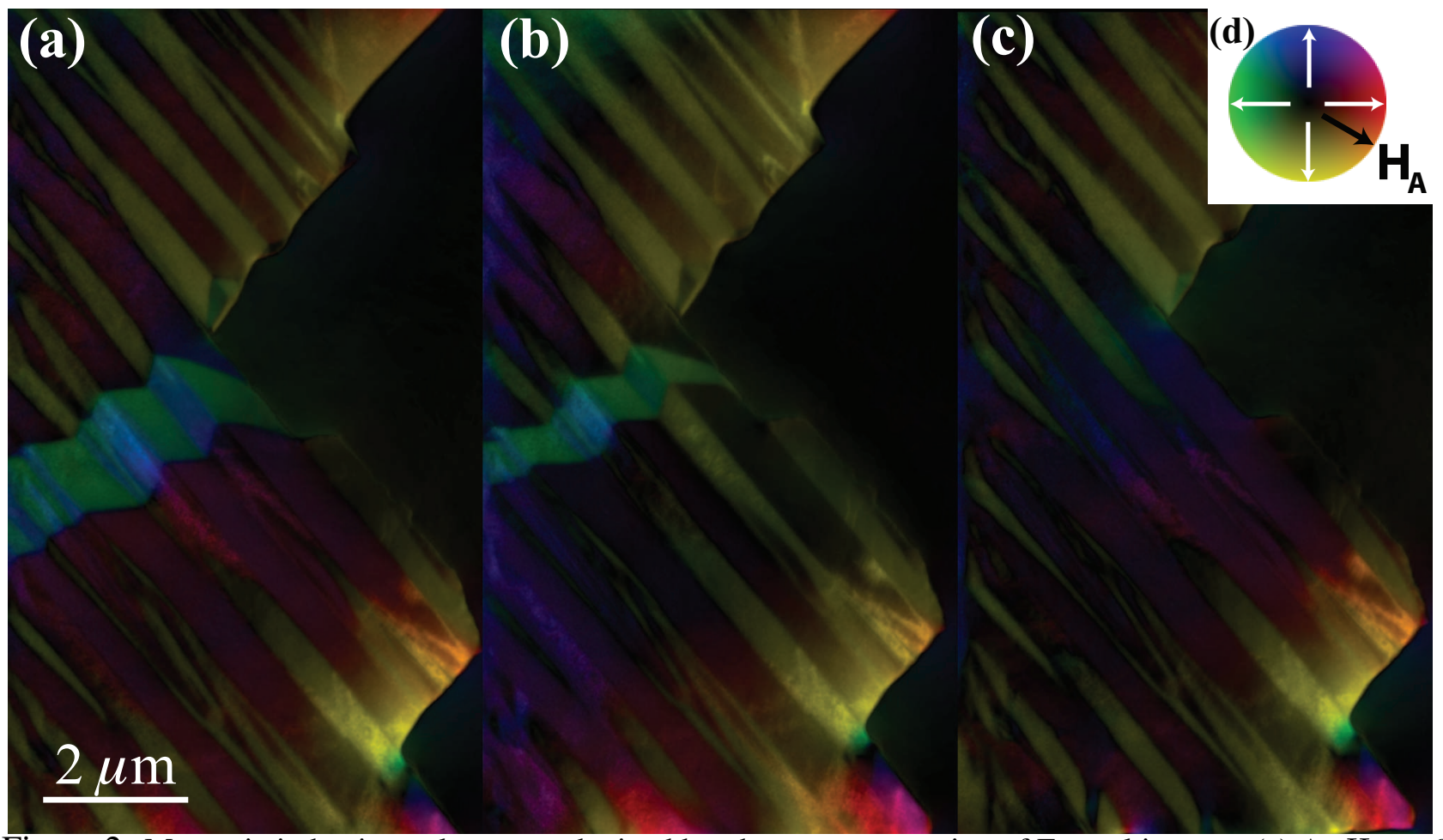

Figure 2: Magnetic induction color maps obtained by phase reconstruction of Fresnel images: (a) At $H_{A}=0$ Oe, (b) $H_{A}=15 \mathrm{Oe}$, (c) $H_{A}=23$ Oe. (d) White arrows in the legend (d) indicate the direction of the induction corresponding to a particular color. The black arrow indicates the direction of the applied field. 